\section{Chimeras are not everything}

Katharina Dittmar

State University of New York-Buffalo, NY, USA

\section{Book review}

Lynn Margulis, Celeste A. Asikainen, Wolfgang E. Krumbein. Eds. Chimeras and Consciousness: Evolution of the Sensory Self. Cambridge, MA: MIT Press; 2011.

The uniting thread of the book is an attempt at an integrated view of the evolution of sensory systems, life, and its consciousness. To accomplish this, the book fuses opinions from different fields of science, and just like the greek mythical fire-breathing chimera, the result is strong, but also impossible.

The book starts out with prototypes of life, and their complex beginnings and interactions. Viruses are introduced as harbingers of selves, as regulators of life and creators of novel genetic combinations. This is followed by an argument for the simultaneous nature of growth processes and early life, contrasting it to the galvanism of the primordial soup theory. Bacterial signaling and sensing is treated as the forerunner of community forming and interaction.

From there, it moves onto examples of how organisms sense, and shape the environment, using microbial groups, human families, and earth as a whole as examples. In particular, it hypothesizes about how communication leads to emergent properties, emphasizing that many designs and skills prescribed as uniquely human predate our existence by eons. Arguing along the lines of the needs of group survival and reproduction, the controversial idea of group selection is once again introduced, this time from a psychology perspective. The all-encompassing finale of this part calls for greater scientific and intellectual awareness of Gaia, as a coherent living entity.

Building on the idea of Gaia a few Chapters treat the interdependencies of cosmic and biological cycles in the development of sensory functions, summarized in an interesting overview of these processes in Chapter 9 (Scofield).

Chimera, part IV, stresses the importance of chimeric unions in the development of life, and sensory interactions - with varying success (see below). Attention is paid to processes of symbiogenesis, as well as chimeric chromosomes. A chimerical origin is invoked for the origin of the immune system.

In its last part, the book moves on to human consciousness, and tries to shed light onto various aspects of brain development and neurobiology, as well as primate communication by olfactory and acoustic signals. In this quest it uses insights from established theory, but also introduces an influence of thermodynamics on choice-making (Sagan, Chapter 21), in perhaps the most challenging Chapter of the book (for scientists and non-scientists alike).

The strength of the book is a pervasive call for more intellectual integration across fields of science, and some Chapters are interesting and informative. Namely, Chapter 19, which deals with a short review of consciousness and cognitive functions in vertebrates. Chapter 12 sheds light on Russian thought and early development on symbiogenesis, but is unfortunately a bit too short. Chapter 5 is a concise argument for bacterial group decision making and its precursor status for more complex cognition.

The book is explicitly compiled to challenge main stream scientific (evolutionary) ideas on multiple levels. Neo-Darwinism seems to be a particular irk of various contributions. In the book's introduction, Margulis frowns at the simplicity of the notion that: '...traits and behaviors of organisms can be well understood simply by focussing on how they benefit the individual, if not directly then in the form of reproductive prowess..', and calling it to be '...put to the test, and abandoned...'. While challenging what is perceived as neo-Darwinistic problems of individualism, overemphasis on competition, and simplicity, various contributors engage in a semantic fallacy, and pick on an extreme (and largely abandoned) interpretation of the theory. More importantly, no relevant alternative and cohesive theory is offered.

Many Chapters are introduced as novel, or innovative, yet some comfortably fit within already established ideas and fields of modern science (e.g., epigenetics; metagenomics; environmental geomicrobiology).

A particular weakness is revealed in Chapter 16 , when principles of the scientific method are completely abandoned for a fictitious account of evolution. Specifically, Vickers and Williamson postulate (with little pertinent evi-
Correspondence: Katharina Dittmar, State University of New York-Buffalo, NY, USA. E-mail: katharinad@gmail.com

Key words: chimera, consciousness, life.

Received for publication: 19 August 2011. Accepted for publication: 6 September 2011.

This work is licensed under a Creative Commons Attribution NonCommercial 3.0 License (CC BYNC 3.0).

(C) Copyright K. Dittmar, 2011

Licensee PAGEPress, Italy

Trends in Evolutionary Biology 2011; 3:e4 doi:10.4081/eb.2011.e4

dence) the evolution of caterpillars by hybridization (hybridogenesis) with velvet worms (Onychophora), resulting in (and explaining) holometabolism.1,2 It is important to be bold, but papers like this open the door to wild speculation, rather than testable hypotheses. Unfortunately, the book often misses its mark of being or initiating a paradigm shift. Not only because it tries to explain everything, while overlooking relevant and integrative recent theoretical, and experimental scientific contributions, but also because some Chapters are lost in complaints against what is perceived as the science establishment (e.g., Chapter 8).

According to the editors, the book aims at readers from the public, as well as the science community. For the uninitiated to science some Chapters may be too dense (e.g., Chapter 21). For scientists, some Chapters may be too short to engage intellectually (e.g., Chapter 1).

In summary, the book is clearly controversial, and thus thought provoking - but the jury is still out on its usefulness.

\section{References}

1. Hart MW, Grosberg RK. Caterpillars did not evolve from onychophorans by hybridogenesis. Proc Natl Acad Sci 2009. [Epub October 30].

2. Giribet G. On velvet worms and caterpillars: Science, fiction, or science fiction? Proc Natl Acad Sci 2009;106:E131. 